\title{
Understanding positive feedback between PNA and synoptic eddies by eddy structure decomposition method
}

\author{
Fang Zhou ${ }^{1} \cdot$ Hong-Li Ren ${ }^{2,3} \cdot$ Xiao-Feng $\mathrm{Xu}^{2} \cdot$ You Zhou $^{1}$
}

Received: 27 December 2015 / Accepted: 28 July 2016 / Published online: 6 August 2016

(C) The Author(s) 2016. This article is published with open access at Springerlink.com

\begin{abstract}
In the upper troposphere during winter, positive synoptic eddy (SE) feedback plays an indispensible role in maintaining the Pacific-North American (PNA) pattern that dominates climate variability on inter-annual timescales over the North Pacific and downstream regions. This study shows that the eddy forcing, induced by eddy-vorticity (EV) fluxes, is not only in-phase with, but also downstream to the PNA pattern in terms of its northeast Pacific lobe. We employ the eddy structure decomposition method to understand such an observed PNA-SEs feedback, and propose a kinematic mechanism that can depict dynamical processes associated with the eddy structure change and its induced positive eddy feedback relative to the PNA flow pattern. With this method, the winter-mean PNA-related SE structures are separated into climatological (basic) and anomalous SE structures, and these two parts can be used to represent the changes in SE structure in a statistical sense and then to calculate the EV fluxes in order to further elucidate the feedback mechanism. It is demonstrated that, on one hand, the winter-mean PNA flow tends to systematically deform the structures of SEs and induce anomalous EV fluxes, and these winter-mean EV fluxes primarily converge into the PNA cyclonic center, which, in return enhances the PNA flow. On the other hand, the PNA-related northeast Pacific flow is featured by a stronger zonal wind shear in
\end{abstract}

Hong-Li Ren

renhl@cma.gov.cn

1 Nanjing University of Information Science and Technology, Nanjing 210044, China

2 Laboratory for Climate Studies, National Climate Center, China Meteorological Administration, 46 Zhongguancun Nandajie St., Haidian District, Beijing 100081, China

3 Joint Center for Global Change Studies, Beijing, China the east than the west, which can induce larger zonal-slanting eddy structure change and then stronger meridional EV fluxes that converge to form downstream feedback. This kinematic mechanism may help to deeply understand the dynamical eddy feedback between the low-frequency PNA flow and high-frequency SEs.

Keywords Pacific-North American (PNA) pattern . Positive eddy feedback · Downstream feedback · Eddy structure decomposition · Meridional eddy fluxes $\cdot$ Zonal eddy fluxes

\section{Introduction}

In the middle-high-latitude atmosphere, synoptic eddy (SE), which usually have a short lifecycle of 2-8 days, and low-frequency (LF) modes, which generally have their lifetime of longer than 2 weeks and always coexist together (Blackmon et al. 1984a, b). The Pacific-North American (PNA) teleconnection pattern is well known to be the prominent LF climate variability in the northern extratropics during wintertime and has large impacts on climate variations over North America (Wallace and Gutzler 1981). The PNA pattern consists of four action centers with alternating signs located over the subtropical northeast Pacific, the Gulf of Alaska, and northwest and southeast North America (e.g., Barnston and Livezey 1987; Chen and van den Dool 2003).

There has been much research into the possible growth mechanisms of LF variability. The first mechanism is direct growth owing to linear dispersion from an external diabatic heating, which has obtained a great success and well recognition worldwide (e.g., Blackmon et al. 1979; Horel and Wallace 1981; Hoskins and Karoly 1981). The second is 
barotropic growth arising from the instability of zonal climatological mean flow asymmetry, which indicates that LF pattern can be generated by atmospheric internal dynamics only without external forcing (e.g., Simmons et al. 1983; Branstator 1992; Kimoto et al. 2001; Feldstein 2002). The third is growth from the dynamical feedback induced by high-frequency SEs (e.g., Lau 1988; Nakamura and Wallace 1993; Branstator 1995; Jin et al. 2006a, b; Luo et al. 2007; Jin 2010; Zhang et al. 2012; Nie et al. 2013; Ren et al. 2014). Many studies have shown that the interactions between synoptic scale eddies and low-frequency flow (referred to as SELF) play an essential role in generating and maintaining the predominant climate modes (e.g., Cai and Mak 1990; Robinson 1991; Lau and Nath 1991; Branstator 1992; Limpasuvan and Hartmann 1999; Lorenz and Hartmann 2003; Jin et al. 2006a; Jin 2010; Ren et al. 2012). On one hand, statistically anomalous SE activity acts to maintain monthly/seasonal mean extratropical climatic variability through upscale energy transport (e.g., Kok et al. 1987; Vautard et al. 1988). On the other hand, LF flow can in return partly modulate the statistically anomalous SE activity, where ambient anomalous mean flow works to systematically organize high-frequency SEs and harvest EV forcing (Nakamura and Wallace 1990; Branstator 1995).

Previous studies on the SELF feedback have usually followed the classical framework of atmospheric climate dynamics that involves interactions among three components of flow: climatological mean flow, LF flow and SE flow. For example, Lau (1988) showed that the in-phase relationship between the anomalous mean flow and the EV forcing induced by EV flux anomalies may yield a positive SELF feedback. However, based on the classic dynamical framework, the dynamical processes by which the LF flow organizes SEs, and the SE structures are changed by LF flow, and then the eddy feedback is induced under the modulation of LF flow, still remain unclear. A limitation of the classic dynamical framework is that the changes in eddy structure are unable to be delineated relative to the climatological-mean SE structure. Therefore, Jin et al. (2006a, b) put forward a new theoretical framework in order to realize a dynamical closure for the two-way interaction between LF flow and SEs through introducing a linear operator into the 'parameterization' of eddy forcing by LF flow itself. Except the complex mathematics, this framework provides a new idea to understand dynamical processes more deeply in the positive eddy feedback on LF flow by statistically separate eddy into its basic part and anomalous part., which represent a climatological measure of SE flow and anomalous SE activity induced by the LF flow, respectively. This new framework includes interactions among four parts: climatological mean flow, LF flow, basic SE flow, and anomalous SE flow. Combining the climatological mean flow with basic SE flow, a linear closure for the dynamical SELF feedback can be derived such that the LF flow can induce the slowly-varying part of SE feedback directly based on a linear operator (Jin et al. 2006a, b; Pan et al. 2006). Further, through the idea of the SE flow separation, Ren et al. (2009) put forward the kinematic mechanism to represent the positive eddy feedback between SEs and North Atlantic Oscillation (NAO) (as LF flow). Meanwhile, a so-called left-hand rule or preference in the SELF feedback has been revealed, i.e., the EV, eddy-moisture, eddy-heat and transformed eddy-potential-vorticity fluxes tend to be directed to the left-hand side of the LF flow (Kug and Jin 2009; Kug et al. 2010a, b, c; Ren et al. 2011).

Note that the EV forcing pattern associated with the winter-mean NAO shows not only an in-phase, but also an upstream feedback onto the NAO pattern. Ren et al. (2012) further followed the basic idea of the framework of Jin et al. (2006a, b) and proposed an eddy structure decomposition method to illustrate how the SEs feedback onto the NAO by examining the SE structure changes induced by NAO and the resultant SE structure anomaly patterns. Under the background NAO flow, the SE structure can be deformed systematically into two kinds of changes: zonal slanting change and meridional shifting change. The former prefers to generate a meridional dipole type of anomalous structure in quadrature with the basic SE, which contributes to generating the net meridional EV fluxes, while the latter generates a meridional dipole type of eddy structure anomalies in phase with the basic SE, contributing to forming the net zonal EV fluxes. Then, in return, the net meridional EV fluxes positively contribute to the NAO flow, and the net zonal fluxes to the upstream enhancement. Furthermore, Ren et al. (2014) developed an empirical eddy-induced growth rate to quantify the efficiency of the eddy feedback as guided by the theory of eddy-induced instability of LF variability proposed by Jin (2010).

Less attention has been paid to the PNA pattern, although it is the dominant mode in the North PacificNorth American sector of the Northern Hemisphere during winter, with two-way interaction with SEs (Lau 1988; Hall and Derome 2000; Rivière and Orlanski 2007). Indeed, previous studies have revealed the key role of the SE feedback as internal dynamics in maintaining the PNA pattern, although the tropical external forcing accompanying events such as El Niño/La Niña is important for PNA excitation (Jin et al. 2006a, b; Pan et al. 2006), following the great circle theory proposed by Hoskins and Karoly (1981). Embedded within the active storm track area of the North Pacific, the PNA has a very distinct spatial feature from that of the NAO (Wallace and Gutzler 1981). Therefore, in this study we will diagnose eddy feedback features associated with PNA and employ the eddy structure decomposition method to explore the physical processes deeply in the dynamical interactions between SEs and the PNA flow, which may 
Fig. 1 Winter-mean PNA index from $1980 / 1981$ to $2009 / 2010$, where linear trend has been removed from the index
Detrended winter PNA index anomalies form 1980/81-2009/10

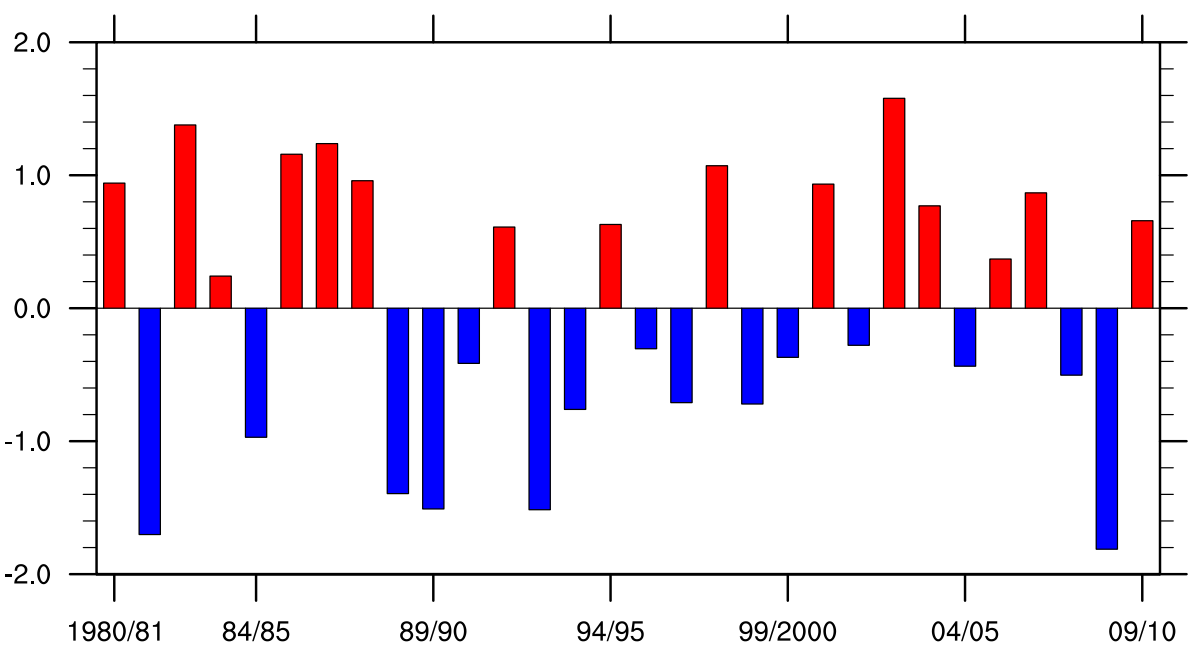

be quite different from those of the NAO. At last, a corresponding kinematic mechanism will be suggested to depict such a positive feedback between SEs and the PNA. This paper is organized as follows. Data and methodology are introduced in Sect. 2. The SE structure and its changes associated with the PNA are presented in Sect. 3. The eddy structure decomposition method is introduced briefly in Sect. 4 and the statistics for eddy feedback associated with the PNA is analyzed in Sect. 5. Mechanisms for eddy feedback under the PNA are identified in Sect. 6 and summary and discussion are given in Sect. 7 .

\section{Data and methodology}

The monthly and daily mean datasets used are from the ERA-Interim reanalysis (Dee et al. 2011). Streamfunction and relative vorticity both are calculated from zonal and meridional winds. The horizontal resolution of the data is $1.5^{\circ} \times 1.5^{\circ}$ and the period is covered from January 1980 to December 2010. Here, a Lanczos filter technique (Duchon 1979 ) is adopted using 41 weights to extract the SE component on the timescale of 2-8 days by band-pass filtering the daily dataset. To avoid boundary effects, a slightly extended range is used when filtering the data. LF variability is simply defined as the winter mean performed for December, January and February (DJF) in each year. Note that the so-called LF eddy with a timescale of about 10-30 days is excluded here because it may not directly contribute to the LF variability (Zhang et al. 2012). All analyses are carried out at the pressure level of $300 \mathrm{hPa}$.

The monthly PNA teleconnection index is directly obtained from the Climate Prediction Center/National Ocean Atmosphere Administration (CPC/NOAA, http://www.cpc. ncep.noaa.gov/products/precip/CWlink/pna/pna.shtml).
Figure 1 shows the detrended winter-mean PNA index calculated by averaging the monthly index over DJF from $1980 / 81$ to $2009 / 10$.

The streamfunction tendency induced by anomalous EV fluxes can be expressed as EV forcing to measure the dynamical SELF feedback. In this paper, the wintermean horizontal EV fluxes and EV forcing are calculated as

$\overline{\mathbf{V}^{\prime} \zeta^{\prime}}=\left(\overline{u^{\prime} \zeta^{\prime}}, \overline{v^{\prime} \zeta^{\prime}}\right)=\mathbf{F}_{r}+\mathbf{F}_{i}$

$\left(\frac{\partial \bar{\psi}}{\partial t}\right)_{\mathrm{se}}=-\Delta^{-1} \nabla \cdot \overline{\mathbf{V}^{\prime} \zeta^{\prime}}$,

respectively, where $u^{\prime}, v^{\prime}$, and $\zeta^{\prime}$ denote the band-passfiltered zonal wind, meridional wind, and vorticity. () denotes the seasonal mean, $\nabla \cdot()$ and $\Delta^{-1}()$ are the horizontal divergence and the horizontal Laplacian inversion operators, respectively, and ()$_{\text {se }}$ indicates the tendency induced by SE. The divergent (convergent) EV fluxes contribute to a positive (negative) tendency of streamfunction, which indicates an anticyclonic (cyclonic) LF vorticity tendency. Here, the EV forcing induced by the EV fluxes is taken as $\mathbf{F}_{i}=-\nabla \cdot{ }^{-1} \nabla \cdot \overline{\mathbf{V}^{\prime} \zeta^{\prime}}$ because only the divergent (irrotational) component of the fluxes can contribute to the LF flow, where $\nabla$ is the horizontal gradient operator.

To clarify the SE structure in the PNA region, we utilize the weighted 3-point covariance method of Ren et al. (2009). In this method, we first choose the main base point near the northeastern Pacific action center of the PNA and also in the Northeast Pacific storm track area, and then calculate the normalized 1-point eddy streamfunction $\left(\psi^{\prime}\right)$ covariance field on $300-\mathrm{hPa}$ level. In this covariance field, two neighboring negative covariance centers can be found in the upstream and downstream. 


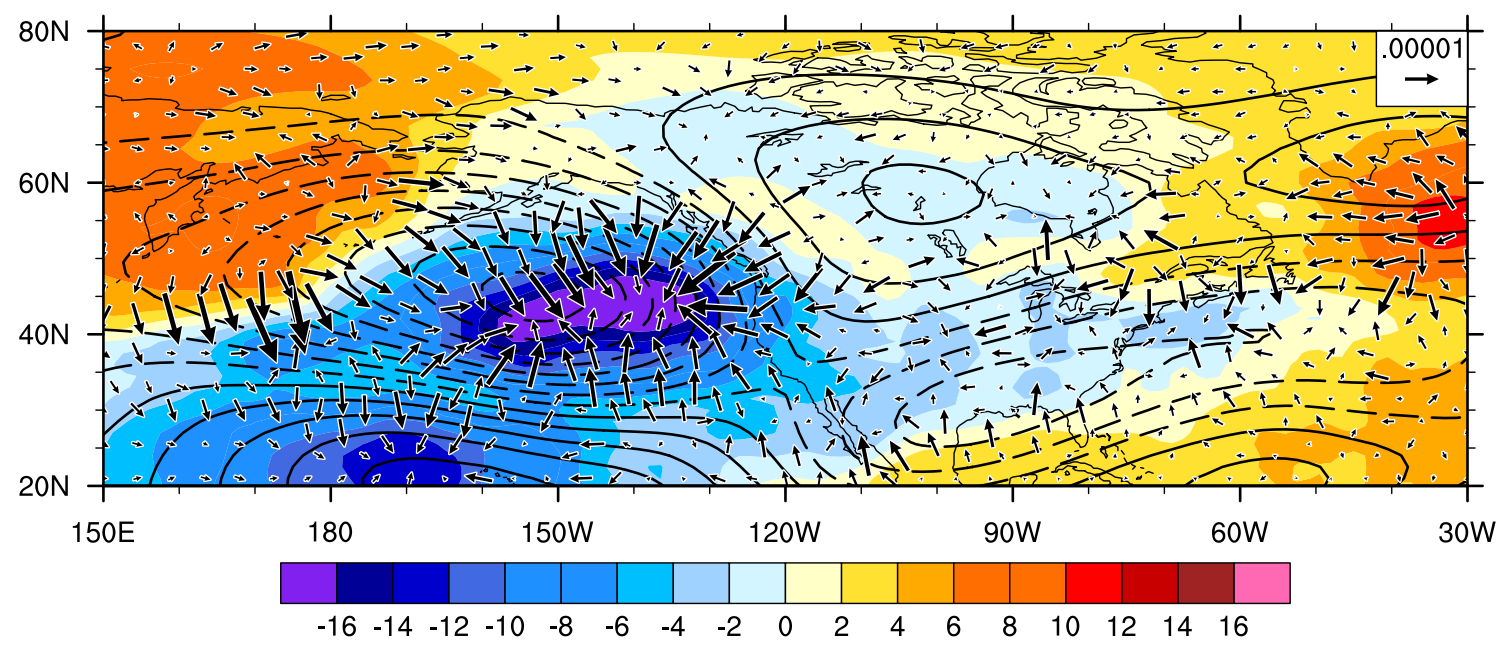

Fig. 2 PNA-index-regressed $300 \mathrm{hPa}$ anomalous streamfunction (contours with an interval of $1 \times 10^{6} \mathrm{~m}^{2} \mathrm{~s}^{-1}$ ), eddy forcing in terms of streamfunction tendency (shading, unit: $\mathrm{m}^{2} \mathrm{~s}^{-2}$ ), and irrotational component of eddy vorticity fluxes (vector, unit: $\mathrm{ms}^{-2}$ )

Following Ren et al. (2009), the normalized field of 1-point covariance for each of the three points is defined as

$$
\begin{aligned}
& \hat{\psi}_{j}\left(x_{j}, y_{j} ; x, y, t_{s}, \tau\right) \\
& \quad={\overline{\psi^{\prime}}\left(x_{j}, y_{j}, t\right) \psi^{\prime}(x, y, t \pm \tau)^{t}}^{t_{s}} / \sigma_{c}\left[\psi^{\prime}\left(x_{j}, y_{j}\right)\right],
\end{aligned}
$$

where $t, x$, and $y$ represents the time-space coordinates. The subscript $j$ is equal to $0,-1$, and 1 , denoting the primary base point and its upstream and downstream points, respectively. Here also $\overline{()}$ represents the DJF mean, in which $t_{s}$ is year, and $\sigma_{c}$ expresses the climatological standard deviation. $\tau$ represents time lag from lead day -2 to 2 in this paper. Then, the weighted average of $\hat{\psi}_{j}$ using these three points can be calculated as

$\hat{\psi}=\frac{1}{2}\left[\hat{\psi}_{0}-\frac{1}{2}\left(\hat{\psi}_{1}+\hat{\psi}_{-1}\right)\right]$.

The field $\hat{\psi}$, defined as statistical eddy streamfunction field, expresses the SE structure and has the physical unit same with streamfunction. After calculating $\hat{\psi}$ in each winter during the data period, a climatological mean can be constructed and then its departures can be defined as the seasonal anomalies of $\hat{\psi}$.

\section{Changes in SE structure associated with the PNA}

Many studies have shown a positive SELF feedback (e.g., Lau 1988), such as the PNA (Kug and Jin 2009), by detecting the association between EV forcing and LF flow patterns. The patterns of EV fluxes and their EV forcing associated with the PNA index are shown in Fig. 2. We can see that strongest EV forcing occurs in the northeastern Pacific cyclone center of the PNA flow with overall the EV fluxes converging. This follows the "left-hand" rule or preference that the EV fluxes tend to be directed into the left-hand side of the LF flow (Kug and Jin 2009; Ren et al. 2009; Kug et al. 2010a, b, c; Ren et al. 2011), essentially suggesting a positive SE feedback. That is, the convergent EV fluxes generate the EV forcing of vorticity with sign opposite to the streamfunction, which enhances the cyclonic circulation in the positive PNA phase, with the reverse also true. Noting that the northeastern Pacific cyclonic circulation is apparently tilted along the northwest-southeast direction, which is largely responsible for much stronger intensity of the PNA-related wind in the east than the west of its center, the regressed EV forcing pattern is not exactly in phase with the PNA pattern, but has a clear downstream shift. Therefore, besides the in-phase eddy feedback, the PNA flow favors an apparent downstream eddy feedback as well, which evidently differs from the upstream feedback of the NAO, as shown by Ren et al. $(2009,2012)$.

To understand the dynamic processes for the positive in-phase and downstream SE feedback, how the EV fluxes and EV forcing patterns are generated under the PNA conditions will be focused on. As mentioned above, the background LF flow can induce deformation of the structure of SEs during their short lifetime. According to Eqs. (3) and (4), we can calculate the 3-point covariance fields with observational data for either under climatological mean or PNA conditions. Noting that the strongest EV forcing occurs in the northeastern Pacific action center of the PNA and is also within the Northeast Pacific storm track area, where SEs are most active and have a relative high eddyinduced growth rate (Ren et al. 2014) than other areas, we choose the base point there. The evolutions of climatological mean and PNA-related SE structures on different 
Fig. 3 Statistical synoptic eddy structure under climatological mean (black contours) and PNA conditions (color shades) from lead day 2 to lag day 2 by using 3-point $\left(43.5^{\circ} \mathrm{N}, 178.5^{\circ} \mathrm{E}\right.$; $46.5^{\circ} \mathrm{N}, 154.5^{\circ} \mathrm{W} ; 40.5^{\circ} \mathrm{N}$, $132^{\circ} \mathrm{W}$ ) covariance field. Anticyclonic (cyclonic) eddy structure patterns are denoted by red shading and solid contours (blue shading and dashed contours) with an interval of $1 \times 10^{6} \mathrm{~m}^{2} \mathrm{~s}^{-1}$. Thick curves are phase lines of the statistical synoptic eddy structures under climatological mean (blue) and PNA conditions (brown). Gray contours denote the PNA pattern, as in Fig. 2
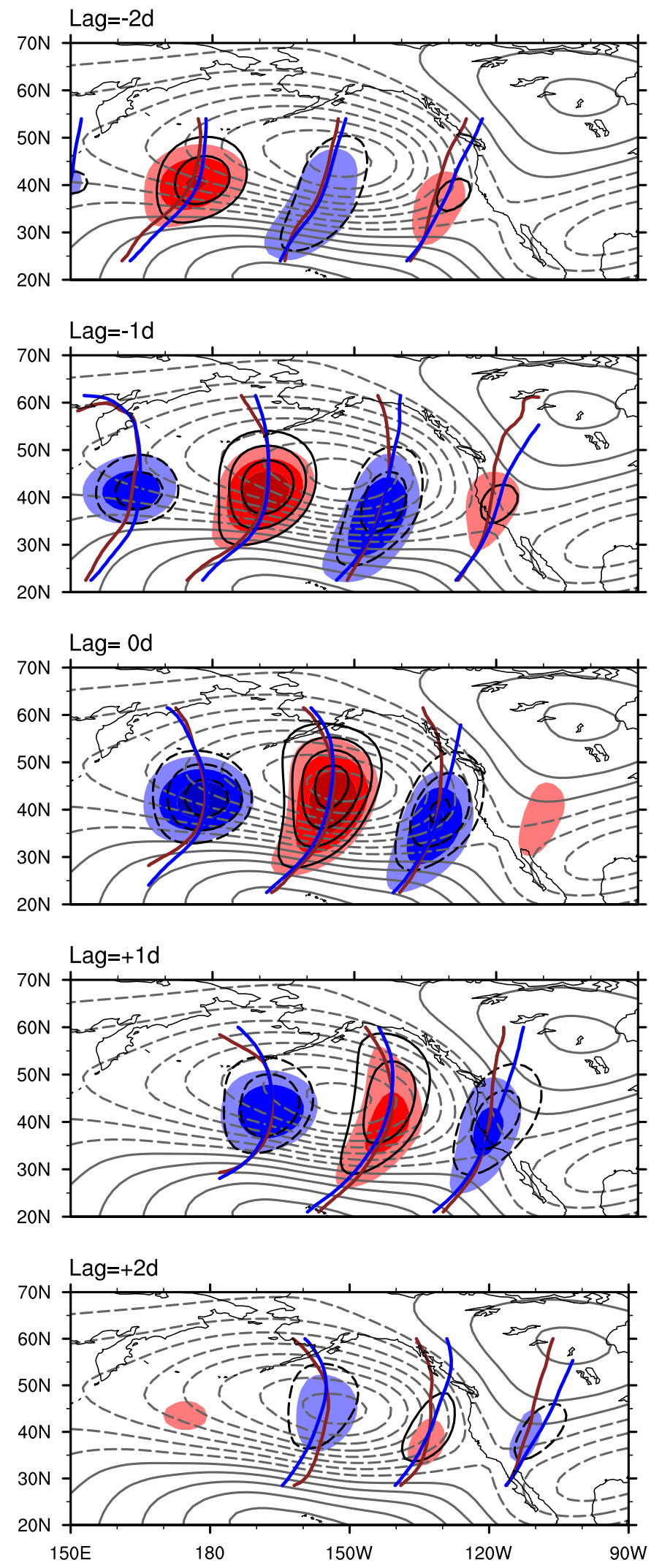

$-5 e+06-4 e+06 \quad-3 e+06-2 e+06 \quad 2 e+06 \quad 3 e+06 \quad 4 e+06 \quad 5 e+06$ 


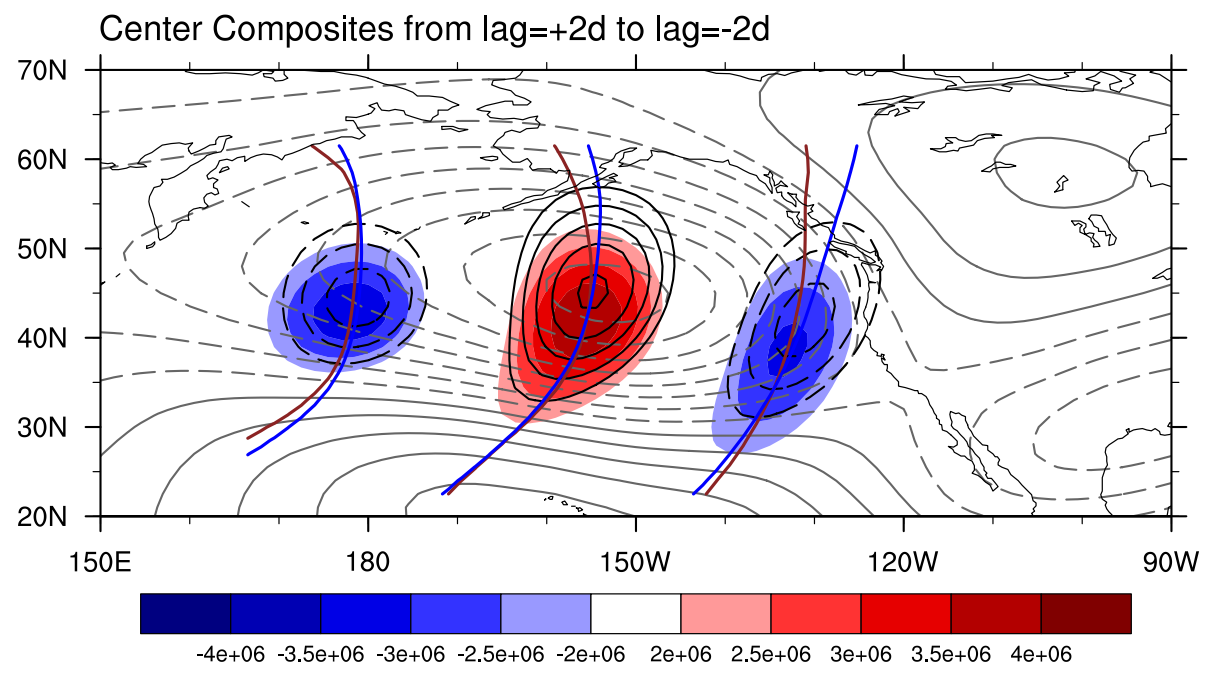

Fig. 4 Center composites for the eddy structure patterns in Fig. 3 generating from lead day 2 to lag day 2 by choosing basic reference points with centers of the central anticyclone at lag 0 in Fig. 3 for the climatological and PNA conditions, respectively. Anticyclonic (cyclonic) eddy structure patterns are denoted by red shading and

lead-lag days are shown in Fig. 3. Firstly, the pattern of climatological SE structure shows a wave-packet-like pattern with alternating signs from west to east, which represents an anticyclonic or cyclonic eddy structure pattern. The packet's amplitude will be clearly gradually decayed with the distance increasing from that main base point. Secondly, it is evident that the circular eddy originates in the upstream area of the northeastern Pacific cyclone center of the PNA, and then gradually intensifies. During its eastward propagating, the SE elongates meridionally, and finally damps downstream the PNA cyclonic center. The amplitude is smaller in the SE-damping area than in the developing area, which is presumably due to the fact that the damping of an eddy is a much faster process than the SE-developing process.

To obtain the PNA-induced SE structure, firstly, the anomalous 3-point covariance field will be regressed onto the winter-mean PNA index. We then multiply this regressed SE structure anomaly pattern by a factor of two and add this inflated pattern to the climatological mean eddy structure pattern. This doubling is only used in Figs. 3 and 4 to illustrate the structure change in terms of the relative changes in the phase lines that are defined simply as the zonal extremum lines of each eddy structure. In Fig. 3, relative to the climatological SE structure, the structure under PNA conditions is deformed systematically into two different types of changes in SE structure, which are associated with wind anomalies of the PNA.

The first type is the zonal-wind shearing effect on the northern and southern flanks of the PNA cyclonic circulation that prefers to make the zonally-slanted SE structure solid contours (blue shading and dashed contours) with an interval of $0.5 \times 10^{6} \mathrm{~m}^{2} \mathrm{~s}^{-1}$. Thick curves are phase lines of the statistical synoptic eddy structures under climatological mean (blue) and PNA conditions (brown). Gray contours denote the PNA pattern, as in Fig. 2

change. When the SEs propagate eastward, the SEs' phase lines incline to be slanted counterclockwise. Because the PNA cyclonic circulation is apparently tilted along the northwest-southeast direction, resulting in much stronger intensity in the east than the west (as seen in the contour density in Fig. 3), the shearing effect of the PNA is stronger to the east. So the angles between phase lines of the PNArelated and climatological mean SE structures are increased as the SEs propagate, which also indicates that the PNA flow acts to change the SE structure by an accumulating effect.

The second type of structural change is caused by the meridional wind in the west of the PNA cyclonic circulation that has a kinematic effect resulting in the eddy structure shifting meridionally southward. In particular, the southward shift of the SE structure appears both in the west and east of the PNA cyclonic circulation region. Noting that the PNA cyclonic circulation features a northwestsoutheast tilt structure, as seen in Fig. 2, the contours of the PNA-related flow are much denser in the east flank and sparser in the west flank. As a result, in the SE-developing region, the anomalous northerly of the PNA has an accelerating effect that starts to shift the eddy structure southward. When the eddy propagates into the damping region, the anomalous southerly of the PNA has a retarding effect that damps the southward acceleration of the eddy, but the eddy structure still remains southward shifting.

To better display the features of eddy structure changes, Ren et al. (2012) proposed a method of center composite, which is quite similar to the averaging during an eddy's life time. It is clear that Fig. 4 is significantly 
characterized by the counterclockwise tilt of the SE's phase lines in the positive PNA phase, relative to the phase lines in the climatological eddy structure pattern. This indicates that the PNA flow contributes to inducing a cyclonic-type slanting SE structure change due to the shearing effect associated with the PNA flow. The angles between phase lines of the PNA-related and climatological mean SE structure are much larger in the downstream area of the PNA cyclonic circulation than in the upstream area due to the asymmetry in the intensity of the PNA flow and the accumulated effect caused by the PNA. In addition, the PNA-related SE structure pattern shows a southward shift, in contrast to the climatology, which is presumably due to the kinematic effect of the background northerly wind anomalies in the west of the PNA cyclonic circulation, and then the eastward propagation of the whole eddy group.

\section{PNA-associated eddy structure decomposition diagnosis}

Jin et al. (2006a) considered that the SE flow is highly chaotic and unpredictable beyond its typical lifecycle. Thus, on a time scale much longer than a week, it may be viewed as stochastic flow. Taking the view that the eddy flow evolution is an assumed stochastic flow ensemble, the time mean is an ergodic approximation that enables us to substitute the ensemble mean. So we can separate the eddy flow into two parts: the basic and anomalous ensembles of the eddy flow. In this study, we take the climatological mean and its departures of the observed eddy flow statistics properties as the basic and anomalous parts of eddy flow.

As the SE structure is modulated from its climatological mean pattern to a zonally slanted and meridionally shifted pattern in the PNA conditions. We then focus on how the SE structure changes feedback positively onto the PNA. Here we follow the method proposed by Ren et al. (2012) to decompose the SE flow structure into basic and anomalous flow structures, and then reconstruct the anomalous EV flux structure. We here introduce this method briefly.

According to Eqs. (3) and (4), the field $\hat{\psi}$ represents the statistical SE structure captured by the normalized covariance pattern and changes from year to year, which can characterize the spatiotemporal evolution the SE structure for each winter. Thus, we can calculate the 3-point covariance field in each winter during the data period, then a climatological mean structure can be constructed as basic SE structure and the regression field on the PNA index is denoted as anomalous SE structure.

Therefore, the SE structure is divided into two parts:

$\hat{\psi}=\hat{\psi}_{c}+\hat{\psi}_{a}$ where the subscripts $c$ and $a$ represents the climatological (basic) and anomalous SE structures, respectively.

As this covariance field is normalized and has the physical unit of streamfunction, the associated pattern of anomalous eddy-velocity and EV structure can be calculated by simply applying the Laplacian operator to the field of anomalous SE structure, as follows

$\hat{\mathbf{V}}=\vec{k} \times \nabla \hat{\psi}$

$\hat{\zeta}=\nabla^{2} \hat{\psi}$

Then, these two fields can be also separated into two parts:

$\hat{\mathbf{V}}=\hat{\mathbf{V}}_{c}+\hat{\mathbf{V}}_{a}$,

$\hat{\zeta}=\hat{\zeta}_{c}+\hat{\zeta}_{a}$

Further, the EV flux structure can be decomposed as

$\hat{\mathbf{V}} \hat{\zeta}=\hat{\mathbf{V}}_{c} \hat{\zeta}_{c}+\hat{\mathbf{V}}_{c} \hat{\zeta}_{a}+\hat{\mathbf{V}}_{a} \hat{\zeta}_{c}+\hat{\mathbf{V}}_{a} \hat{\zeta}_{a}$

Therefore, we can divide the EV flux structure into four different parts as follows: the basic eddy velocity multiplied by basic eddy vorticity (BB), the basic eddy velocity multiplied by anomalous eddy vorticity (BA), the anomalous eddy velocity multiplied by basic eddy vorticity $(\mathrm{AB})$, and the anomalous eddy velocity multiplied by anomalous eddy vorticity (AA). Considering $\hat{\mathbf{V}}_{a} \hat{\zeta}_{a}$ is a nonlinear small term which will be detailedly revealed in Sect. 5 , we can approximate the anomalous eddy flux by $(\hat{\mathbf{V}} \hat{\zeta})_{a} \approx \hat{\mathbf{V}}_{c} \hat{\zeta}_{a}+\hat{\mathbf{V}}_{a} \hat{\zeta}_{c}$, where $\hat{\mathbf{V}}_{a} \hat{\zeta}_{a}$ has been neglected. In the following section, the two linear components (BA and $\mathrm{AB}$ ) of $(\hat{\mathbf{V}} \hat{\zeta})_{a}$ will be examined.

\section{Anatomy of eddy feedback}

Here, we anatomize the each big anomalous term in eddy feedback through examining the anomalous eddy structure changes and the induced anomalous eddy-vorticity fluxes under the organization of the PNA flow in order to deeply understand the dynamical processes in generating the positive in-phase and downstream feedback of SEs onto the PNA flow.

We first examine the PNA-related BA pattern, $\hat{\mathbf{V}}_{c} \hat{\zeta}_{a}$. As seen in Fig. 5a, on the west side of the PNA cyclonic circulation, the negative (positive) EV anomalies appear centered in the upper (lower) part of the basic cyclonic eddy structure. But in the center and on the east side, the positive (negative) EV anomalies occur in the upper middle-right of the anticyclone (cyclone) and lower middle-right of the cyclone (anticyclone) in the basic eddy-structure pattern. Based on the anomalous EV structure and the basic eddyvelocity structure patterns (viz., $\hat{\zeta}_{a}$ and $\hat{\mathbf{V}}_{c}$ ), the anomalous 
Fig. 5 a Center composites covariance fields for statistical synoptic eddy structure under climatological mean $\left(\hat{\psi}_{c}\right.$, contours with an interval of $\left.1 \times 10^{6} \mathrm{~m}^{2} \mathrm{~s}^{-1}\right)$ and anomalous eddy vorticity structure $\left(\hat{\zeta}_{a}\right.$, shading, unit: $\mathrm{s}^{-1}$ ) under PNA conditions. b Anomalous eddy vorticity fluxes $\left(\hat{\mathbf{V}}_{c} \hat{\zeta}_{a}\right.$, black vectors, obtained by averaging the vector fields from lead 2 days to lag 2 days including zero lag, unit: $\mathrm{ms}^{-2}$ ) and the induced eddy forcing (shading, unit: $\mathrm{m}^{2} \mathrm{~s}^{-2}$ ). Black contours denote the PNA pattern, as in Fig. 2
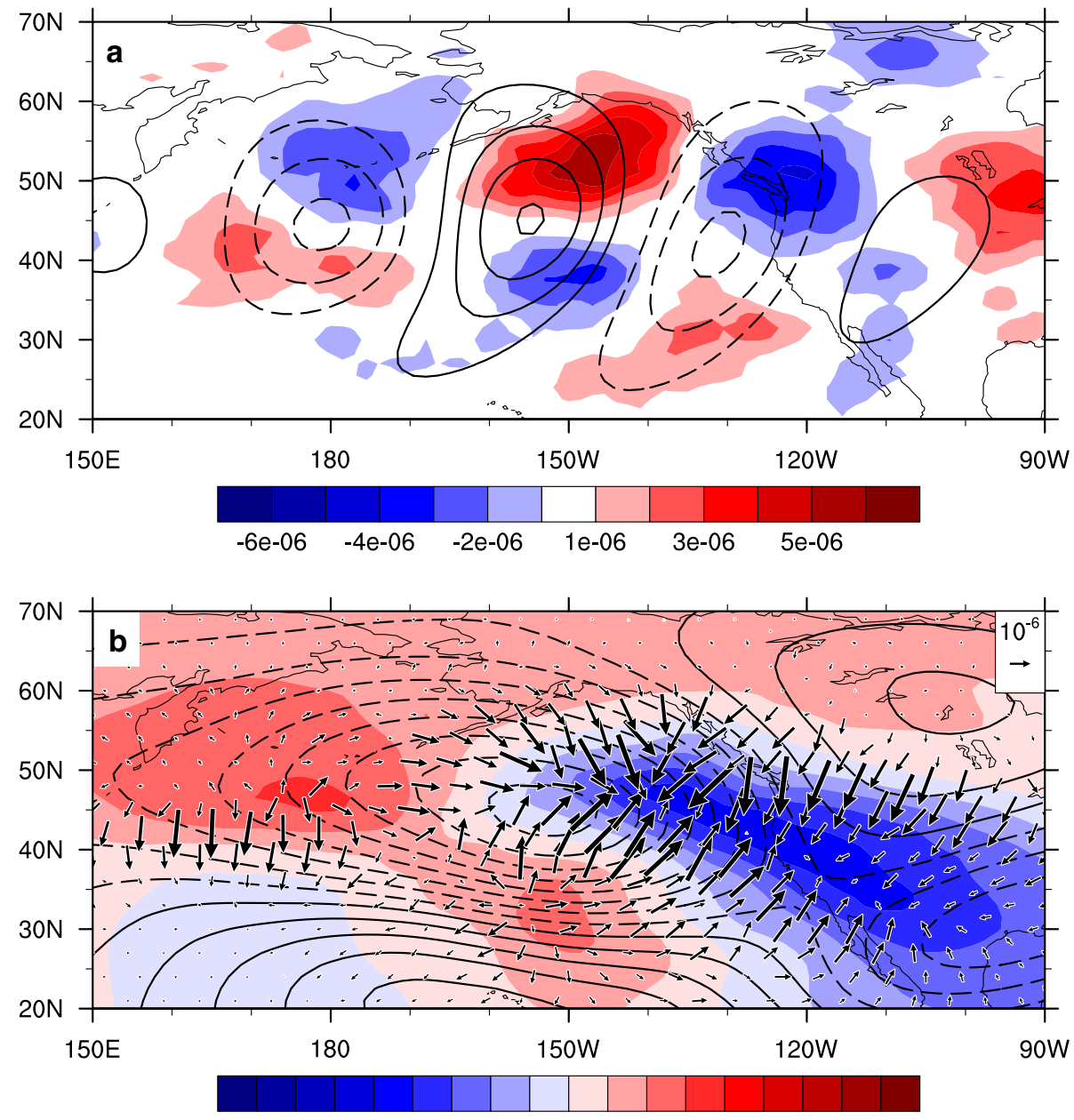

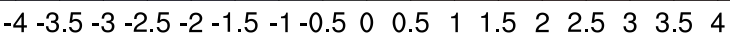

EV fluxes (viz. $\hat{\mathbf{V}}_{c} \hat{\zeta}_{a}$ ) related with the PNA can be calculated directly (Fig. 5b) with rotational component removed.

The reconstructed EV flux anomalies are clearly dominated by their meridional component and tend to follow the left-hand rule in the center and east side of the PNA cyclonic circulation, with strong equatorward fluxes on the northern flank of the PNA and poleward fluxes on the southern flank. However, the meridional fluxes on the west side of the PNA are opposite with slight poleward fluxes on the northern flank of the PNA and equatorward fluxes on the southern flank. Except for these meridional EV fluxes, the zonal EV fluxes with smaller magnitude are also evident on the east and west of the PNA cyclonic center, where the PNA-related meridional winds are relatively large and thus partly contribute to the zonal component of EV fluxes. Overall, the convergence of EV flux anomalies occurs in the center and east of the PNA cyclonic circulation and the divergence does in the west, leading to strong positive vorticity tendency induced by SEs over the center and east, and slight negative vorticity tendency to the west, which can be seen clearly in the EV forcing pattern in Fig. 5 b.
To examine the PNA-related AB pattern, viz. $\hat{\mathbf{V}}_{a} \hat{\zeta}_{c}$, Fig. $6 \mathrm{a}$ shows the basic EV structure and the anomalous eddy-velocity structure patterns $\left(\hat{\zeta}_{c}\right.$ and $\left.\hat{\mathbf{V}}_{a}\right)$. These two patterns are somewhat similar to those in Fig. 5a, but with clear differences. There are positive (negative) eddy streamfunction anomalies in the lower (upper) part of the basic negative EV structure within the PNA region. In contrast, there are negative (positive) eddy streamfunction anomalies in the lower (upper) part of the basic positive EV structure. As shown in Fig. 6b, the major characteristics in the $\mathrm{AB}$ pattern are generally opposite compared with Fig. 5b, but its magnitudes are smaller, as well as the zonal component more clearly dominates the EV fluxes over the PNA region, compared with the dominance of the meridional component in Fig. 5b. It can be seen in Fig. 6b that, in the middle of the basic EV structure, all the zonal fluxes tend to be westward because $\hat{\mathbf{V}}_{a}$ is westward (eastward) and $\hat{\zeta}_{c}$ is positive (negative) over the basic cyclonic (anticyclonic) eddy structure. Further, these zonal fluxes converge in the west and diverge in the east of the PNA region, giving an upstream eddy feedback, similar to the NAO (Ren et al. 2012), which can be clearly seen in the EV forcing pattern. 
Fig. 6 a Center composites covariance fields for statistical synoptic eddy vorticity structure under climatological mean $\left(\hat{\zeta}_{c}\right.$, shading, unit: $\left.\mathrm{s}^{-1}\right)$ and anomalous eddy structure $\left(\hat{\psi}_{a}\right.$, contours with an interval of $2 \times 10^{5} \mathrm{~m}^{2} \mathrm{~s}^{-1}$ ) under PNA conditions. b Climatological mean eddy vorticity fluxes $\left(\hat{\mathbf{V}}_{a} \hat{\zeta}_{c}\right.$, black vectors, obtained by averaging the vector fields from lead 2 days to lag 2 days including zero lag, unit: $\mathrm{ms}^{-2}$ ) and the induced eddy forcing (shading, unit: $\mathrm{m}^{2} \mathrm{~s}^{-2}$ ). Black contours denote the PNA pattern, as in Fig. 2
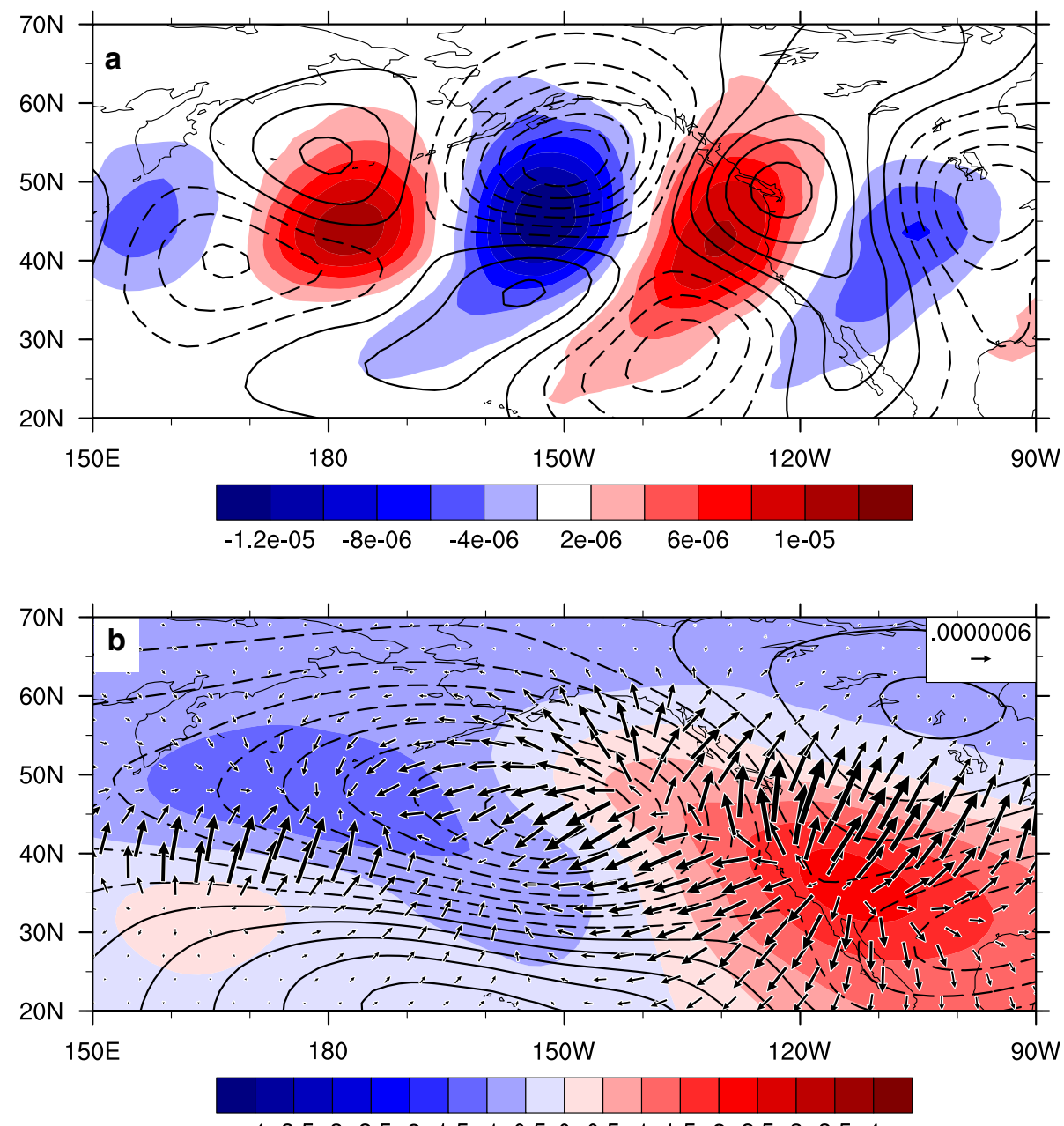

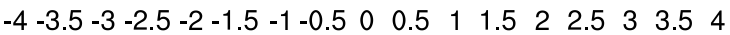

To illustrate that the nonlinear term, viz. $\hat{\mathbf{V}}_{a} \hat{\zeta}_{a}$, is a relative small term in Eq. (10), we here also examine the PNArelated AA pattern which is shown in Fig. 7. In Fig. 7a we can see that the anomalous EV structure and the anomalous eddy-velocity structure patterns $\left(\hat{\zeta}_{a}\right.$ and $\left.\hat{\mathbf{V}}_{a}\right)$ are almost in phase. As expected, the anomalous EV fluxes and induced EV forcing related with the PNA shown in Fig. $7 \mathrm{~b}$ are fairly much smaller relative to the $\mathrm{BA}$ and $\mathrm{AB}$ patterns. Therefore, the $\mathrm{BA}$ and $\mathrm{AB}$ patterns are the big terms that mainly contribute to the anomalous EV fluxes. The magnitudes of the BA fluxes are larger than those of the AB fluxes. In Fig. 8, the distribution of integrated $\hat{\mathbf{V}}_{c} \hat{\zeta}_{a}+\hat{\mathbf{V}}_{a} \hat{\zeta}_{c}$ EV fluxes are generally similar to those in Fig. 2, where the zonal EV fluxes cancel each other out along the central latitudes of PNA due to the comparable BA and AB components. All the anomalous EV fluxes act to be convergent into the PNA cyclonic circulation. Accordingly, there is a positive vorticity tendency induced by SEs over the central cyclonic circulation in the PNA pattern and thus enhances the PNA flow. Moreover, the meridional fluxes are much larger in the east of the PNA region and give greater convergence, viz., EV forcing, which is downstream of the center of the cyclonic circulation of PNA and quite consistent with that in Fig. 2.

\section{Kinematic mechanism for eddy feedback under PNA}

As seen in Figs. 5 and 6, over the PNA cyclonic region, the BA pattern inclines to induce meridional EV fluxes with larger magnitude than zonal fluxes, whereas the $\mathrm{AB}$ pattern favors larger zonal EV fluxes. These features can be seen more clearly in the individual components of eddy fluxes, as shown in Fig. 9. In fact, the patterns seen in the observations in Fig. 2 are a combination of the $\mathrm{BA}$ and $\mathrm{AB}$ flux patterns. Here, we propose a kinematic mechanism for the PNA-related eddy feedback, shown in schematic form in Fig. 10.

Without loss of generality, we take a LF cyclonic circulation into account as representing the primary action center located over the northeastern Pacific in the positive 
Fig. 7 a Center composites covariance fields for anomalous synoptic eddy vorticity structure $\left(\hat{\zeta}_{a}\right.$, shading, unit: $\left.\mathrm{s}^{-1}\right)$ and anomalous eddy structure $\left(\hat{\psi}_{a}\right.$, contours with an interval of $2 \times 10^{5} \mathrm{~m}^{2} \mathrm{~s}^{-1}$ ) under PNA conditions. b Anomalous eddy vorticity fluxes $\left(\hat{\mathrm{V}}_{\mathrm{a}} \hat{\zeta}_{\mathrm{a}}\right.$, black vectors, obtained by averaging the vector fields from lead 2 days to lag 2 days including zero lag, unit: $\mathrm{ms}^{-2}$ ) and the induced eddy forcing (shading, unit: $\mathrm{m}^{2} \mathrm{~s}^{-2}$ ). Black contours denote the PNA pattern, as in Fig. 2

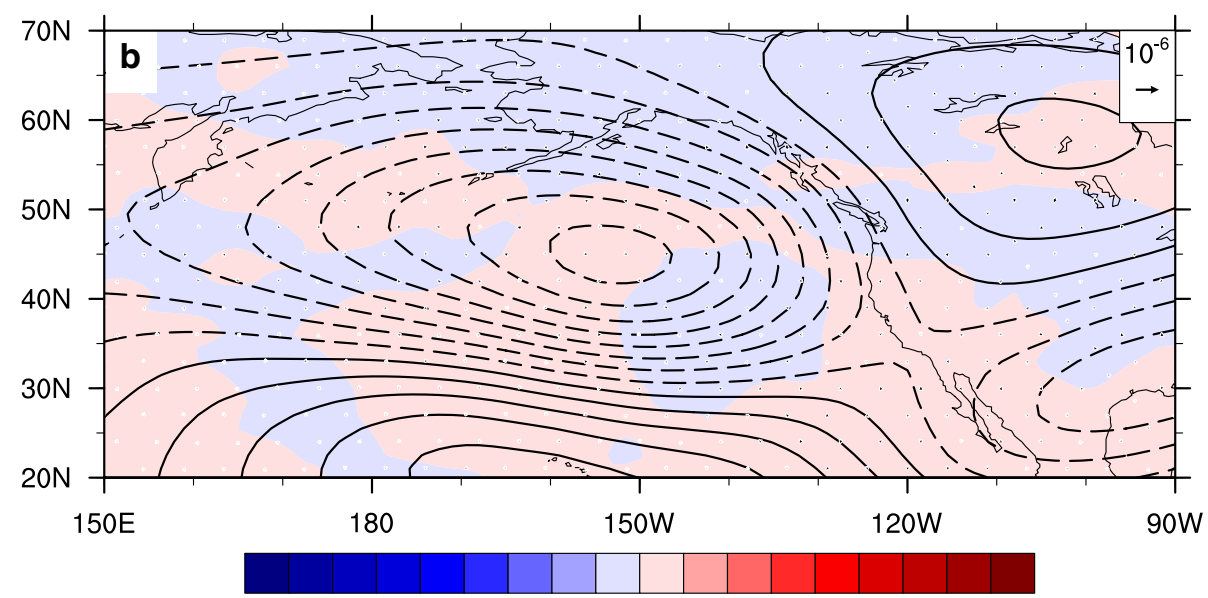

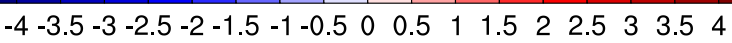
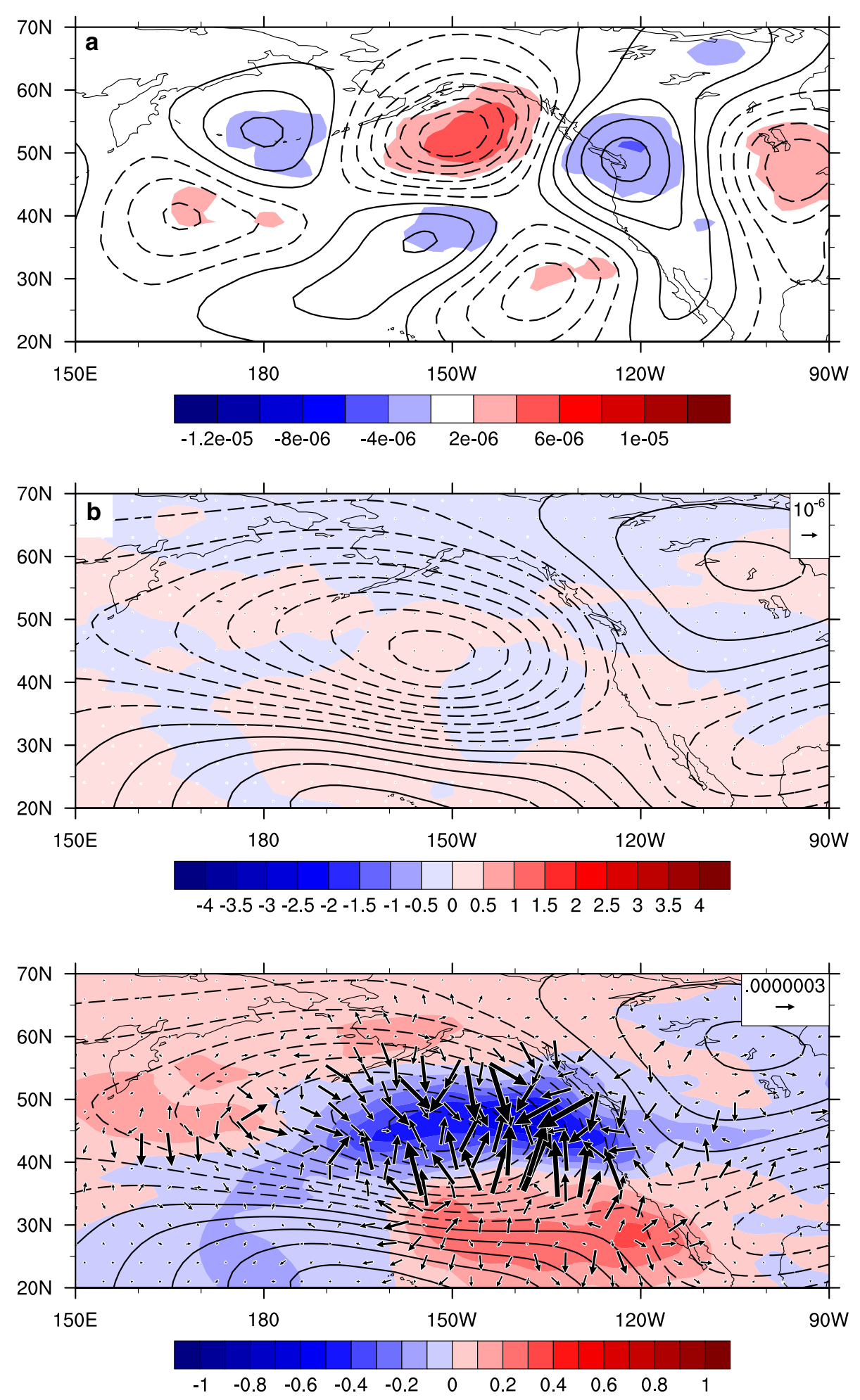

Fig. 8 The integrated eddyvorticity fluxes in terms of $\hat{\mathbf{V}}_{c} \hat{\zeta}_{a}+\hat{\mathbf{V}}_{a} \hat{\zeta}_{c}$ (black vectors obtained by averaging the vector fields from lead 2 days to lag 2 days including zero lag, unit: $\mathrm{ms}^{-2}$ ) and the induced eddy forcing (shading, unit: $\mathrm{m}^{2} \mathrm{~s}^{-2}$ ). Black contours denote the PNA pattern
PNA phase. Essentially, the dynamic process for the SE feedback is a mixture of the two dominant linear parts (BA and AB). As suggested by Ren et al. (2012), the barotropic part of the anomalous SE structures is a domination of two idealized patterns in general: the meridional in-quadrature dipole (IQD, see Fig. 10a, d) and the meridional in-phase dipole (IPD, see Fig. 10b, e), which are both relative to basic SE structure. All the observed anomalous SE structure patterns can be obtained by combining the IQD and IPD patterns with different phases. Further, based on the observational diagnoses, we consider four idealized eddy structure patterns: BA IQD (Fig. 10a), BA IPD (Fig. 10b), 

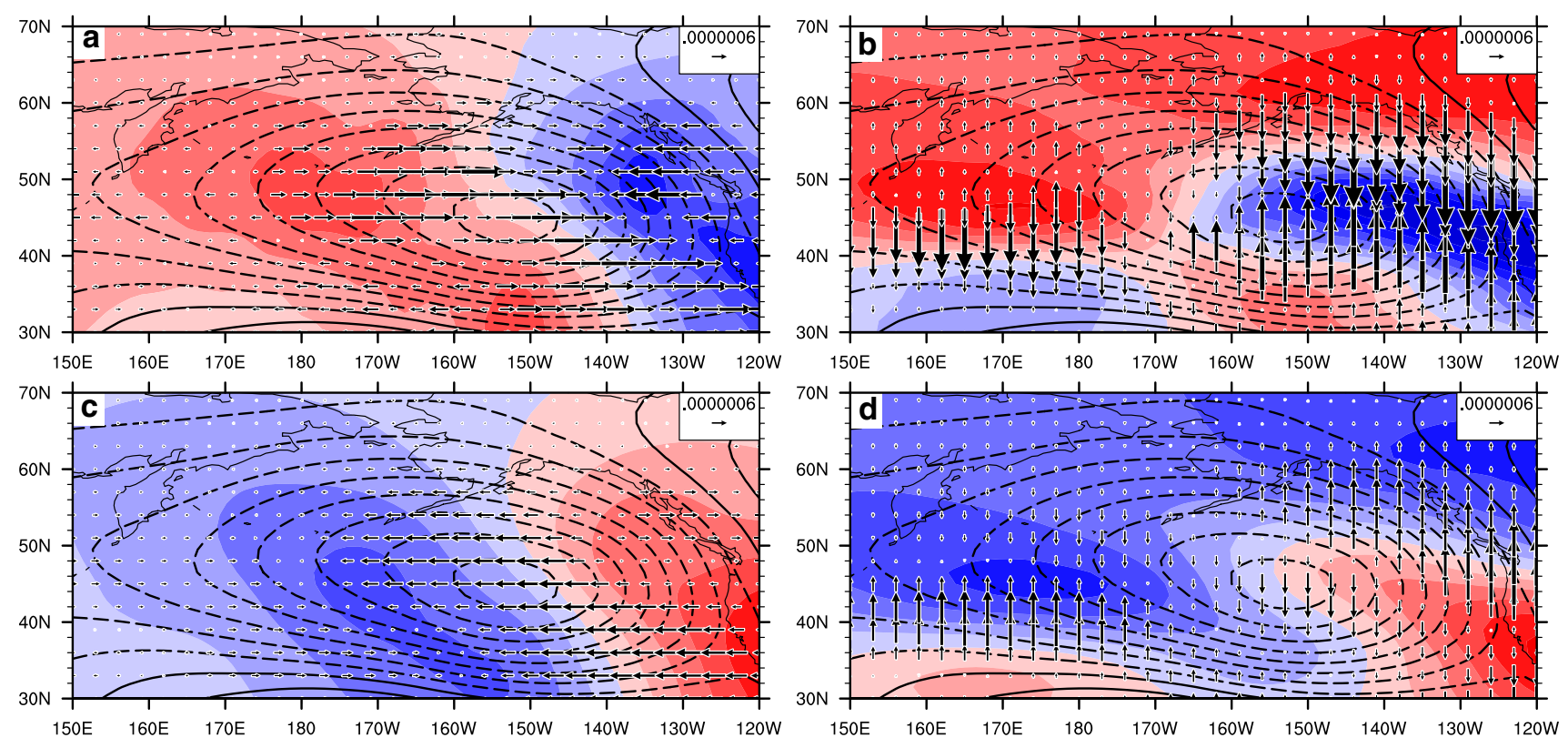

$\begin{array}{llllllllllllllllllllllll}-2 & -1.8 & -1.6 & -1.4 & -1.2 & -1 & -0.8 & -0.6 & -0.4 & -0.2 & 0 & 0.2 & 0.4 & 0.6 & 0.8 & 1 & 1.2 & 1.4 & 1.6 & 1.8 & 2\end{array}$

Fig. 9 Eddy vorticity fluxes $\hat{\mathbf{V}}_{c} \hat{\zeta}_{a}(\mathbf{a}, \mathbf{b})$ and $\hat{\mathbf{V}}_{a} \hat{\zeta}_{c}$ (c, d), where a, $\mathbf{c}$ are for zonal component and $\mathbf{b}, \mathbf{d}$ for meridional component. The black vectors are obtained by averaging the vector fields from lead

AB IQD (Fig. 10d), and AB IPD (Fig. 10e), as set up by the eddy streamfunction and $\mathrm{EV}$ fields.

SEs (cyclone/anticyclone) that passing through the cyclonic LF flow region are zonally slanted during their relatively short lifetime by the barotropic shearing effect of the background PNA flow. Therefore, SE structures are tilted counterclockwise relative to the basic case, with negative (positive) vorticity anomalies in the upper left and lower right of the basic anticyclonic (cyclonic) eddy structures, which favors a meridional BA IQD structure. As shown in Fig. 10a, $\hat{\mathbf{V}}_{c}$ and $\hat{\zeta}_{a}$ are in quadrature. In the northern upstream (downstream) of the PNA cyclonic circulation, $\hat{v}_{c}>0\left(\hat{v}_{c}<0\right)$ and $\hat{\zeta}_{a}<0\left(\hat{\zeta}_{a}>0\right)$, while in the southern upstream (downstream) of the PNA cyclonic circulation, $\hat{v}_{c}>0\left(\hat{v}_{c}<0\right)$ and $\hat{\zeta}_{a}>0\left(\hat{\zeta}_{a}<0\right)$. Thus, the calculated BA fluxes tend to be equatorward $\left(\hat{v}_{c} \hat{\zeta}_{a}<0\right)$ on the northern flank of the PNA cyclonic center and poleward $\left(\hat{v}_{c} \hat{\zeta}_{a}>0\right)$ on the southern flank. This IQD-type eddy structure can generate the meridional BA fluxes that are convergent into the LF cyclonic circulation.

With the kinematic effect of the background northerly wind anomalies in the west of the PNA cyclonic circulation, the SEs show a meridionally southward shift compared with the basic structure. So we can see positive (negative) $\mathrm{EV}$ anomalies in the upper part of a basic anticyclonic (cyclonic) eddy structure and lower part of a basic cyclonic (anticyclonic) eddy structure, forming a meridional IPD
2 days to lag 2 days including zero lag (unit: $\mathrm{ms}^{-2}$ ) and the shading is the induced eddy forcing (unit: $\mathrm{m}^{2} \mathrm{~s}^{-2}$ ). Black contours denote the PNA pattern, as in Fig. 2

pattern of BA structure. As shown in Fig. 10b, $\hat{\mathbf{V}}_{c}$ and $\hat{\zeta}_{a}$ are in phase. In the upper (lower) part of a basic anticyclonic (cyclonic) eddy structure, $\hat{u}_{c}>0$ and $\hat{\zeta}_{a}>0$, while in the lower (upper) part of a basic anticyclonic (cyclonic) eddy structure, $\hat{u}_{c}<0$ and $\hat{\zeta}_{a}<0$. Thus, the calculated BA fluxes tend to be eastward $\left(\hat{u}_{c} \hat{\zeta}_{a}>0\right)$. Besides, in the upper left and lower right of a basic cyclonic (anticyclonic) eddy structure, $\hat{v}_{c} \hat{\zeta}_{a}>0$, while in the upper right and lower left of a basic cyclonic (anticyclonic) eddy structure, $\hat{v}_{c} \hat{\zeta}_{a}<0$. Thus, the meridional fluxes with almost opposite signs cancel each other out for an eddy propagating eastward. So this IPD-type eddy structure can produce the eastward zonal BA fluxes throughout the PNA cyclonic circulation.

Similarly, in the AB IQD pattern (Fig. 10d), $\hat{\mathbf{V}}_{a}$ and $\hat{\zeta}_{c}$ are in quadrature. In the upper part of a basic anticyclonic (cyclonic) EV structure, $\hat{v}_{a}<0 \quad\left(\hat{v}_{a}>0\right)$ and $\hat{\zeta}_{c}<0\left(\hat{\zeta}_{c}>0\right)$, while in the lower part of a basic anticyclonic (cyclonic) EV structure, $\hat{v}_{a}>0\left(\hat{v}_{a}<0\right)$ and $\hat{\zeta}_{c}>0$ $\left(\hat{\zeta}_{c}<0\right)$. Thus, the calculated meridional $\mathrm{AB}$ fluxes tend to be poleward $\left(\hat{v}_{a} \hat{\zeta}_{c}>0\right)$ on the northern flank of the PNA cyclonic circulation, and equatorward $\left(\hat{v}_{a} \hat{\zeta}_{c}<0\right)$ on the southern flank. This IQD-type structure can generate the meridional $\mathrm{AB}$ fluxes that diverge from the center of cyclonic LF flow. The AB IPD pattern is shown in Fig. 10e, where $\hat{\mathbf{V}}_{a}$ and $\hat{\zeta}_{c}$ are in phase. In the middle of the basic anticyclonic (cyclonic) EV structure, $\hat{u}_{a}>0 \quad\left(\hat{u}_{a}<0\right)$, which gives westward zonal AB fluxes. Because the $\hat{u}_{a}$ and 
a idealized BA IQD-type structure

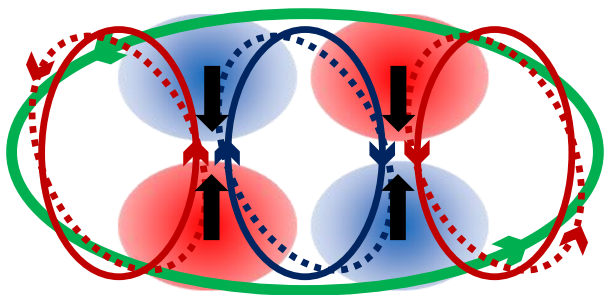

b idealized BA IPD-type structure

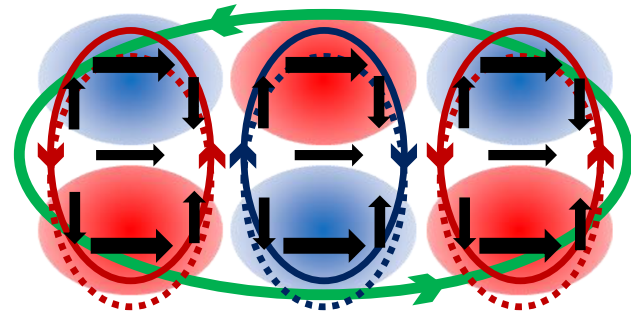

C PNA BA structure

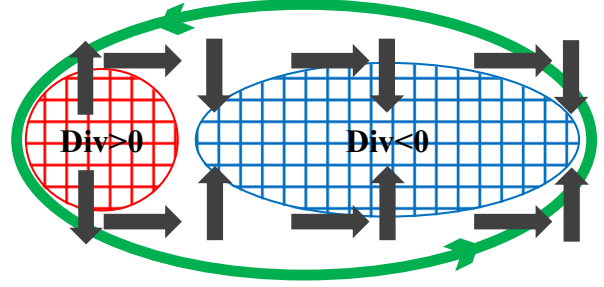

Fig. 10 Schematic diagrams for synoptic eddy feedback onto lowfrequency mode. $\mathbf{a}, \mathbf{b}$ The idealized IQD and IPD-type anomalies for BA structures. $\mathbf{c}$ PNA BA structure. d, e The idealized IQD and IPDtype anomalies for $\mathrm{AB}$ structures. $\mathrm{f}$ PNA $\mathrm{AB}$ structure. Thick green solid ellipses with counterclockwise arrows represent cyclonic low frequency flow (like the north pacific action center) of the positive phase PNA flow. a, b Solid thin circles indicate the idealized synoptic eddy patterns with cyclonic (red) and anticyclonic (blue) eddies, whereas dashed circles denote the changed eddy structures as a result

$\hat{\zeta}_{c}$ are much larger in the center of the basic eddy, the zonal westward fluxes are much stronger in the middle of the PNA cyclonic circulation than on either side. So the zonal AB fluxes will converge upstream and diverge downstream, which indicates an upstream feedback.

We may combine the effect of idealized BA IQD and IPD patterns and generate a PNA-related BA eddy flux pattern (Fig. 10c) to represent the observed BA flux pattern features associated with the PNA. Due to the PNA cyclonic circulation is apparently tilted along the northwest-southeast direction, resulting in much stronger intensity in the east than the west, the shearing effect of background zonal wind in the PNA is stronger in the east. The PNA-associated eddy structures tilt more strongly in the downstream area than in the upstream area, which enables to induce stronger meridional BA fluxes downstream than upstream. d idealized AB IQD-type structure

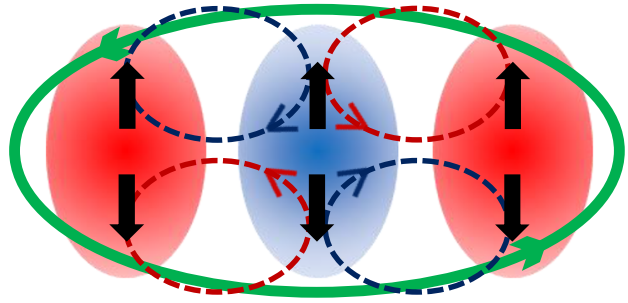

e idealized AB IPD-type structure

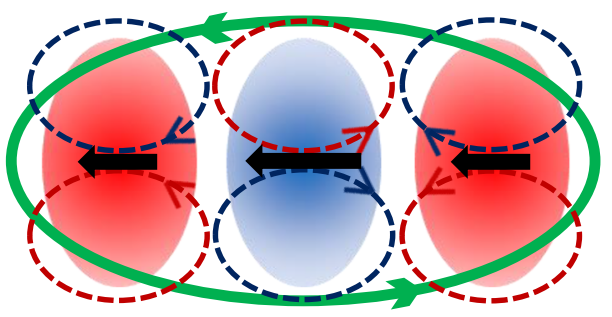

f PNA $\mathrm{AB}$ structure

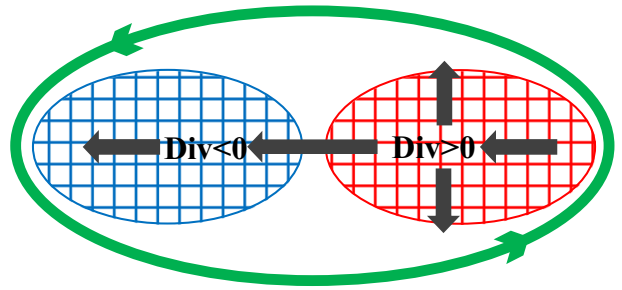

of the anomalous climatological flow. d, e Dashed circles stand for the anomalous eddy flow structures with cyclonic (red) and anticyclonic (blue) circulations. a, b, d, e Small (big) shaded ovals stand for the anomalous (climatological) eddy vorticity, where red is positive and blue is negative. Black solid arrows denote the eddy vorticity fluxes. c, f Dark gray solid arrows denote the net eddy vorticity fluxes and the blue (red) grid represents their convergence (divergence)

Owning to the anomalous northerly of background PNA flow, the PNA-associated SE structure patterns shift meridionally southward, which can induce consistent eastward BA fluxes. Meridional BA fluxes with larger magnitude than the zonal fluxes (seen in Fig. 9a, b) mainly converge in the center and east of the PNA cyclonic circulation and slightly diverge over the west of the PNA, and therefore can induce an EV forcing pattern downstream of the PNA circulation pattern, as shown in Fig. $5 \mathrm{~b}$, contributing to maintenance of the PNA.

Similarly, we can obtain the PNA-related AB eddy flux pattern by combining the effect of idealized AB IQD and IPD patterns. As seen in Fig. 10f, since the zonal westward fluxes are much stronger in the middle of the PNA cyclonic circulation than on either side, and the meridional fluxes in the downstream area tend to be poleward on the 
Fig. 11 Zonal and meridional components of the integrated eddy fluxes of $\hat{\mathbf{V}}_{c} \hat{\zeta}_{a}+\hat{\mathbf{V}}_{a} \hat{\zeta}_{c}$ (black vectors, obtained by averaging the vector fields from lead 2 days to lag 2 days including zero lag, unit: $\mathrm{ms}^{-2}$ ) and the induced eddy forcing (shading, unit: $\mathrm{m}^{2} \mathrm{~s}^{-2}$ ). Black contours denote the PNA pattern

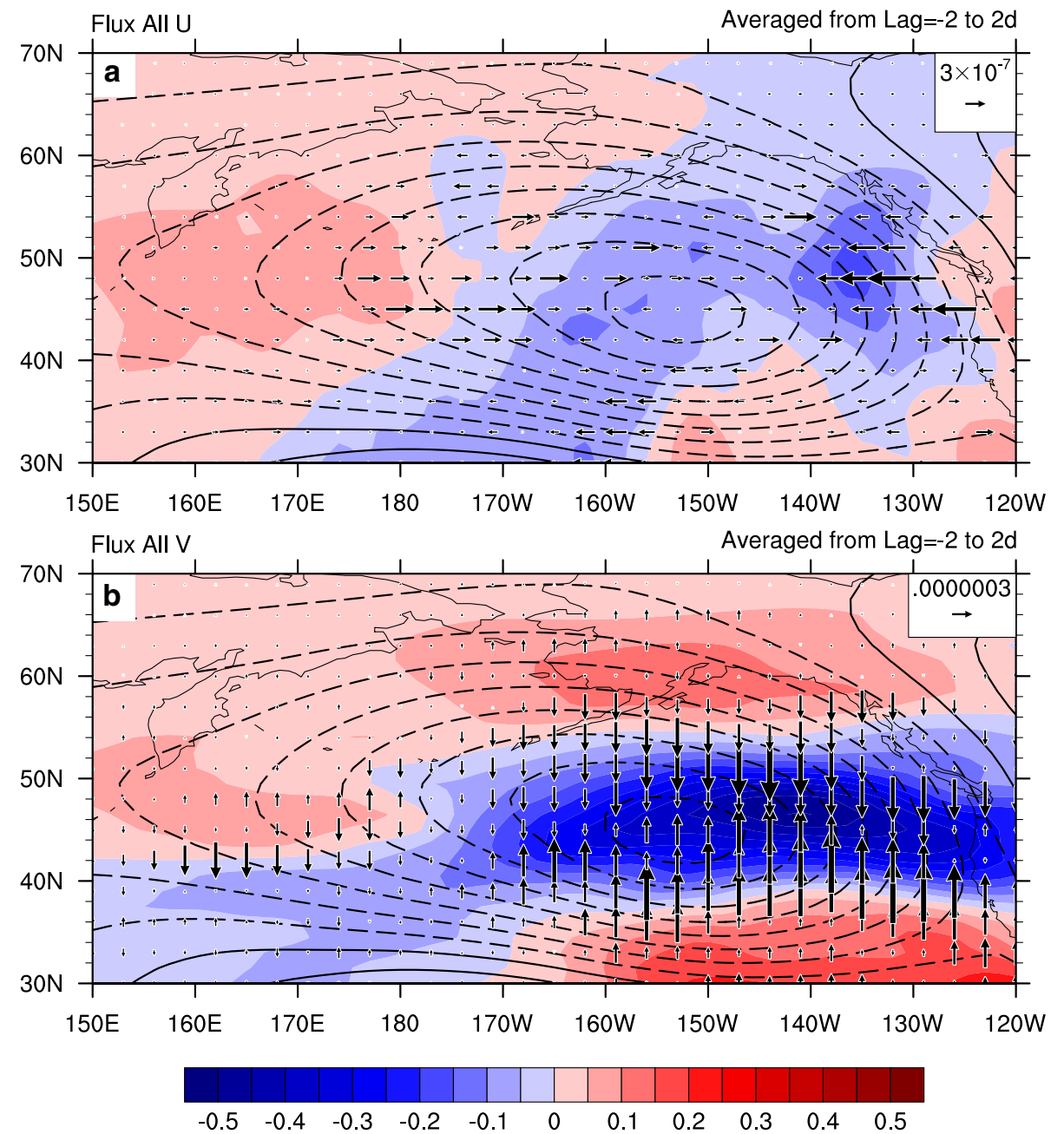

northern flank and equatorward on the southern flank, the PNA-related AB fluxes will converge upstream and diverge downstream, which is similar to that in Fig. 6.

Considering the combination of Fig. 10c, $\mathrm{f}$, all the EV flux anomalies tend to converge into the cyclonic circulation in the PNA pattern (as seen in Fig. 8) and enhance the PNA flow, whereas the fluxes do not give a clear convergence upstream of the PNA circulation (see Fig. 2), unlike the NAO case (Ren et al. 2012). In Fig. 8 we can see that both the zonal and meridional fluxes tend to induce a downstream EV forcing pattern. We integrate the zonal and meridional fluxes of $\hat{\mathbf{V}}_{c} \hat{\zeta}_{a}+\hat{\mathbf{V}}_{a} \hat{\zeta}_{c}$ (see Fig. 11), and find that the meridional fluxes have larger magnitude than the zonal ones and are much stronger downstream. This may presumably be because the PNA cyclonic circulation is much more intense in the east than in the west, so the shearing effect of the PNA is much greater downstream. Therefore, the meridional BA fluxes caused by the BA IQD pattern are much stronger downstream, which results in the downstream feedback. This can also be interpreted as the downstream area being the SE-damping region, where the damping of SEs is conducive to release energy and then is easy to generate a positive feedback.

\section{Summary and discussion}

Using the ERA-Interim reanalysis data, this study shows that the eddy forcing, induced by EV fluxes, is not only positively in-phase with, but also downstream to the northeast Pacific lobe of the PNA pattern and further propose a kinematic mechanism to explain such a positive feedback between the PNA pattern and SEs, using the eddy structure decomposition method developed by Ren et al. (2012). Based on the theoretical framework of Jin et al. (2006a, b), structures of the SE flow can be separated into the basic part and its corresponding anomalous part. The SE structure under the PNA conditions, which is constructed with the 3-point fields of covariance of the SE streamfunction, has been separated into two components: the basic eddy structure pattern and the anomalous eddy structure pattern as modulated by the PNA flow, which can be considered to physically represent the climatological 
and anomalous parts of SE flow in theory, respectively, suggested by Ren et al. (2009, 2012). The two components of SE structure can be utilized to calculate the EV fluxes approximately from the observations and then depict the dynamic process of the PNA related SELF feedback.

Regressed EV forcing pattern shows that the maximum positive SE feedback region occurs in the storm track regions in the north Pacific, where the SEs are most active and have a relatively high eddy-induced growth rate (Ren et al. 2014). Noting that the EV forcing matches the Northeast Pacific and the southeast North America action centers, but does not match the subtropical northeast Pacific and northwest North America action centers. This does not mean that the PNA is only a tropical forcing triggered by Rossby wave-train and SE feedback is unnecessary in maintaining the PNA. Actually, the positive SE feedback in the Northeast Pacific action center works to maintain the PNA pattern. SE structure patterns clearly show that in the positive PNA phase, when the SE structure passing through the cyclonic LF circulation, it undergoes a counterclockwise tilt and a southward shift, relative to the climatology. Based on the eddy structure decomposition method with the nonlinear term neglected, the dynamical processes of how the SE structure changes feed back positively onto the PNA can be clearly illustrated. The tilt, related to the PNAinduced shearing effect, leads to the IQD-type SE structure anomalies in the $\mathrm{BA}$ and $\mathrm{AB}$ patterns that are to large degree responsible for generating the meridional EV fluxes. The shift leads to the IPD-type SE structure anomalies in the $\mathrm{BA}$ and $\mathrm{AB}$ patterns, which preferentially contribute to generating zonal EV fluxes. By integrating the BA and $\mathrm{AB}$ patterns under the PNA conditions, the dynamical SE feedback and the net EV flux anomaly patterns agree well with the observed patterns. It is worth mentioning that this kinematic mechanism is quasi-linear assumed, some highly nonlinear event is not considered in it. As some recent studies (e.g., Nie et al. 2014) suggest that the wave breaking which results in strong potential vorticity filamentation is an important process at least for the LF dipolar mode. Thus, the roles of highly nonlinear events in the maintenance of PNA flow need further investigation in the future.

Previously, Lau (1988) demonstrated that there is a positive SELF feedback over the northern Pacific and Atlantic regions. Further inference can be made that such a kinematic mechanism of positive eddy feedback should apply to more general LF flow modes not only the PNA and NAO, but also the AO and AAO, which can be further explored. However, the PNA has a significant zonally distributed pattern with four major nodes, which is quite distinct from the NAO, that is characterized by a meridional structure. In particular, the primary northeastern Pacific action center of the PNA is tilted along the northwest-southeast direction, and the intensity of the cyclonic circulation is much stronger in the east than the west, which makes the shearing effect much more notable downstream of the PNA cyclonic circulation. Therefore, the relative displacement between the PNA-associated and climatological phase lines grows as the SEs propagate downstream. As the tilt of the eddy structure is more pronounced downstream than upstream, the BA IQD anomalous structure pattern works to induce much stronger meridional BA fluxes and then EV forcing in the downstream area, which forms a downstream feedback onto the PNA pattern. Indeed, the downstream area of the PNA (rather than the upstream area) is also where the SEs usually damp and thus release their energy, which may contribute to a positive feedback downstream. But, due to the PNA pattern features different structures and intensities in each winter, what role the downstream feedback plays in shaping the specific PNA pattern is still unknown. Furthermore, it is also not clear whether the downstream feedback contributes to the PNA pattern variations from season to season. Therefore, the role of the downstream eddy feedback in forming the dynamics of PNA flow is still unclear to some degree and requires further study.

Acknowledgments This work was jointly supported by National Science Foundation (NSF) Grant of China (Grant No. 41375062), the National Basic Research Program of China (973 Program, Grant No. 2015CB453203), and the China Meteorological Special Project (Grant No. GYHY201406022). The authors especially appreciate the three anonymous reviewers for their invaluable comments and suggestions.

Open Access This article is distributed under the terms of the Creative Commons Attribution 4.0 International License (http://creativecommons.org/licenses/by/4.0/), which permits unrestricted use, distribution, and reproduction in any medium, provided you give appropriate credit to the original author(s) and the source, provide a link to the Creative Commons license, and indicate if changes were made.

\section{References}

Barnston AG, Livezey RE (1987) Classification, seasonality and persistence of low-frequency atmospheric circulation patterns. Mon Weather Rev 115:1083-1126. doi:10.1175/1520-0493(1987)115<1083:CSAPOL> 2.0.CO; 2

Blackmon ML, Madden RA, Wallace JM, Gutzler DS (1979) Geographical variations in the vertical structure of geopotential height fluctuations. J Atmos Sci 36:2450-2466. doi:10.1175/1520-0469(1979)036<2450:GVITVS>2.0.CO;2

Blackmon ML, Lee YH, Wallace JM (1984a) Horizontal structure of 500mb height fluctuations with long, intermediate and short time scales. J Atmos Sci 41:961-979. doi:10.1175/1520-0469(1984)041<0961:HSO $\mathrm{MHF}>2.0 . \mathrm{CO} ; 2$

Blackmon ML, Lee YH, Wallace JM, Hsu HH (1984b) Time variation of $500 \mathrm{mb}$ height fluctuations with long, intermediate and short time scales as deduced from lag-correlation statistics. J Atmos Sci 41:981-991. doi:10.1175/1520-0469(1984)041<0981:TVO $\mathrm{MHF}>2.0 . \mathrm{CO} ; 2$ 
Branstator G (1992) The maintenance of low-frequency atmospheric anomalies. J Atmos Sci 49:1924-1945. doi:10.1175/1520-0469(1992)049<1924:TMOLFA >2.0.CO;2

Branstator G (1995) Organization of storm track anomalies by recurring low-frequency circulation anomalies. J Atmos Sci 52:207-226. doi:10.1175/1520-0469(1995)052<0207:OOSTAB >2.0.CO;2

Cai M, Mak M (1990) Symbiotic relation between planetary and synoptic-scale waves. J Atmos Sci 47:2953-2968. doi:10.1175/1520-0469(1990)047<2953:SRBPAS>2.0.CO;2

Chen WY, van den Dool HM (2003) Sensitivity of teleconnection patterns to the sign of their primary action center. Mon Weather Rev 131:2885-2899. doi:10.1175/1520-0493(2003)131<2885:SOTP TT>2.0.CO;2

Dee DP, Uppala SM, Simmons AJ et al (2011) The ERA-Interim reanalysis: configuration and performance of the data assimilation system. Quart J R Meteor Soc 137:553-597. doi:10.1002/qj.828

Duchon CE (1979) Lanczos filtering in one and two dimensions. J Clim 18:1016-1022. doi:10.1175/1520-0450(1979)018<1016:LFIOA $\mathrm{T}>2.0 . \mathrm{CO} ; 2$

Feldstein SB (2002) Fundamental mechanisms of the growth and decay of the PNA teleconnection pattern. Quart J R Meteor Soc 128:775-796. doi:10.1256/0035900021643683

Hall NMJ, Derome J (2000) Transience, nonlinearity, and eddy feedback in the remote response to El Niño. J Atmos Sci 57:3992-4007. doi:10.1175/1520-0469(2001)058<3992:TNAEFI >2.0.CO;2

Horel JD, Wallace JM (1981) Planetary-scale atmospheric phenomena associated with the southern oscillation. Mon Weather Rev 109:813-829. doi:10.1175/1520-0493(1981)109<0813:PSAPA $\mathrm{W}>2.0 . \mathrm{CO} ; 2$

Hoskins BJ, Karoly DJ (1981) The steady linear response of a spherical atmosphere to thermal and orographic forcing. J Atmos Sci 38:1179-1196. doi:10.1175/1520-0469(1981)038<1179:TSLRO $\mathrm{A}>2.0 . \mathrm{CO} ; 2$

Jin FF (2010) Eddy induced instability for low-frequency variability. J Atmos Sci 67:1947-1964. doi:10.1175/2009JAS3185.1

Jin FF, Pan LL, Watanabe M (2006a) Dynamics of synoptic eddy and low-frequency flow interaction. Part I: a linear closure. J Atmos Sci 63:1677-1694. doi:10.1175/JAS3715.1

Jin FF, Pan LL, Watanabe M (2006b) Dynamics of synoptic eddy and low-frequency flow interaction. Part II: a theory for lowfrequency modes. J Atmos Sci 63:1695-1708. doi:10.1175/ JAS3716.1

Kimoto M, Jin FF, Watanabe M, Yasutomi N (2001) Zonal flow-eddy coupling and a neutral mode theory for Arctic Oscillations. Geophys Res Lett 28:737-740. doi:10.1029/2000GL012377

Kok CJ, Opsteegh JD, van den Dool HM (1987) Linear models: useful tools to analyze GCM results. Mon Weather Rev 115:19962008. doi:10.1175/1520-0493(1987)115<1996:LMUTTA>2.0. $\mathrm{CO} ; 2$

Kug JS, Jin FF (2009) Left-hand rule for synoptic eddy feedback on low-frequency flow. Geophys Res Lett. doi:10.1029/200 8GL036435

Kug JS, Jin FF, Park JH, Ren HL, Kang IS (2010a) A general rule for synoptic-eddy feedback onto low-frequency flow. Clim Dyn 35:1011-1026. doi:10.1007/s00382-009-0606-8

Kug JS, Jin FF, Park JH, Ren HL (2010b) Role of synoptic eddies on low-frequency precipitation variation. J Geophys Res 115:D19115. doi:10.1029/2009JD013675

Kug JS, Choi DH, Jin FF, Kwon WT, Ren HL (2010c) Role of synoptic eddy feedback on polar climate responses to the anthropogenic forcing. Geophys Res Lett 37:L14704. doi:10.1029/201 0GL043673

Lau NC (1988) Variability of the observed midlatitude storm tracks in relation to low-frequency changes in the circulation pattern. J Atmos Sci 45:2718-2743. doi:10.1175/1520-0469(1988)045<2718:VOT $\mathrm{OMS}>2.0 . \mathrm{CO} ; 2$
Lau NC, Nath MJ (1991) Variability of the baroclinic and barotropic transient eddy forcing associated with monthly changes in the midlatitude storm tracks. J Atmos Sci 48:2589-2613. doi:10.1175/1520-0469(1991)048<2589:VOTBAB>2.0.CO;2

Limpasuvan V, Hartmann DL (1999) Eddies and the annular modes of climate variability. Geophys Res Lett 26:3133-3136. doi:10.102 9/1999GL010478

Lorenz DJ, Hartmann DL (2003) Eddy-zonal flow feedback in the Northern Hemisphere winter. J Clim 16:1212-1227. doi:10.1175/1520-0442(2003)16<1212:EFFITN>2.0.CO;2

Luo D, Lupo AR, Wan H (2007) Dynamics of eddy-driven low-frequency dipole modes. Part I: a simple model of North Atlantic Oscillations. J Atmos Sci 64:3-28. doi:10.1175/JAS3818.1

Nakamura H, Wallace JM (1990) Observed changes in baroclinic wave activity during the life cycles of low-frequency circulation anomalies. J Atmos Sci 47:1100-1116. doi:10.1175/1520-0469(1990)047<1100:OCIBWA >2.0.CO;2

Nakamura H, Wallace JM (1993) Synoptic behavior of baroclinic eddies during the blocking onset. Mon Weather Rev 121:18921903. doi:10.1175/1520-0493(1993)121<1892:SBOBED>2.0. $\mathrm{CO} ; 2$

Nie Y, Zhang Y, Yang XQ, Chen G (2013) Baroclinic anomalies associated with the Southern Hemisphere Annular Mode: roles of synoptic and low-frequency eddies. Geophys Res Lett 40:2361-2366. doi: $10.1002 / g r l .50396$

Nie Y, Zhang Y, Yang XQ, Chen G, Yang XQ (2014) Quantifying barotropic and baroclinic eddy feedbacks in the persistence of the Southern Annular Mode. Geophys Res Lett 41:8636-8644. doi:10.1002/2014GL062210

Pan LL, Jin FF, Watanabe M (2006) Dynamics of synoptic eddy and low-frequency flow interaction. Part III: baroclinic model results. J Atmos Sci 63:1709-1725. doi:10.1175/JAS3717.1

Ren HL, Jin FF, Kug JS et al (2009) A kinematic mechanism for positive feedback between synoptic eddies and NAO. Geophys Res Lett 36:L11709. doi:10.1029/2009GL037294

Ren HL, Jin FF, Kug JS, Gao L (2011) Transformed eddy-PV flux and positive synoptic eddy feedback onto low-frequency flow. Clim Dyn 36:2357-2370. doi:10.1007/s00382-010-0913-0

Ren HL, Jin FF, Gao L (2012) Anatomy of synoptic Eddy-NAO interaction through eddy structure decomposition. J Atmos Sci 69:2171-2191. doi:10.1175/JAS-D-11-069.1

Ren HL, Jin FF, Kug JS (2014) Eddy-induced growth rate of lowfrequency variability and its mid-late winter suppression in the northern hemisphere. J Atmos Sci 71:2281-2298. doi:10.1175/ JAS-D-13-0221.1

Rivière G, Orlanski I (2007) Characteristics of the Atlantic stormtrack eddy activity and its relation with the North Atlantic Oscillation. J Atmos Sci 64:241-266. doi:10.1175/JAS3850.1

Robinson WA (1991) The dynamics of low-frequency variability in a simple model of the global atmosphere. J Atmos Sci 48:429-441. doi:10.1175/1520-0469(1991)048<0429:TDOLFV>2.0.CO;2

Simmons AJ, Wallace JM, Branstator GW (1983) Barotropic wave propagation and instability, and atmospheric teleconnection patterns. J Atmos Sci 40:1363-1392. doi:10.1175/1520-0469(1983)040<1363:BWPAIA > 2.0.CO;2

Vautard R, Legras B, Déqué M (1988) On the source of midlatitude low-frequency variability. Part I: a statistical approach to persistence. J Atmos Sci 45:2811-2843. doi:10.1175/1520-0469(1988)045<2811:OTSOML >2.0.CO;2

Wallace JM, Gutzler DS (1981) Teleconnections in the geopotential height field during the North Hemisphere winter. Mon Weather Rev 109:784-812. doi:10.1175/1520-0493(1981)109<0784:TIT $\mathrm{GHF}>2.0 . \mathrm{CO} ; 2$

Zhang Y, Yang X, Nie Y, Chen G (2012) Annular mode like variation in a multi-layer QG model. J Atmos Sci 69:2940-2958 